\title{
ESTIMACIÓN DE LA CALIDAD DE SITIO MEDIANTE «ÍNDICES DE SITIO»PARA Cedrelinga catenaeformis Ducke (Tornillo) EN PLANTACIONES DE JENARO HERRERA, LORETO (PERÚ)
}

\author{
Erasmo Otárola*, Luis Freitas*, Carlos Linares*, Juan Baluarte*
}

\section{RESUMEN}

En el área de influencia del Centro de Investigaciones Jenaro Herrera (CIJH), se realizó la estimación de la calidad de sitio a partir de «índices de sitio» para la especie tornillo, Cedrelinga catenaeformis Ducke. Dicha estimación se llevó a cabo a través de la evaluación de 1349 árboles, que comprende mediciones multitemporales de 29 parcelas permanentes de crecimiento. Cada una de ellas contiene entre 25 y 49 árboles distribuidos en 15 plantaciones forestales experimentales.

Se construyeron curvas de índices de sitio utilizando la altura dominante de las parcelas a diferentes edades, que van desde 0,3 hasta 27 años. La edad base elegida fue de 15 años por ser la más representativa en la base de datos. Desarrollando la metodología descrita por Clutter et al. (1983), se ajustaron las curvas de índices de sitio.

Para estimar el comportamiento de la altura dominante en relación con la edad, se utilizó el modelo de Schumacher $\left.[\mathrm{Ln}(\mathrm{Hd})]=b_{1}+b_{2}(1 / \mathrm{E})^{\mathrm{k}}\right]$; donde: $\mathrm{Ln}=$ Logaritmo natural, $b_{1}$ y $b_{2}=$ coeficientes y $\mathrm{k}=$ constante. Para construir la ecuación que estima el índice de sitio con base en la edad y la altura dominante, se utilizó el método de la curva guía, descrito por Hugell (1991). Así se simplificó la ecuación anterior: Ln(IS)= $\mathrm{Ln}(\mathrm{Hd})+\mathrm{b}\left(1 / \mathrm{Eb}^{\mathrm{k}}-1 / \mathrm{E}^{\mathrm{k}}\right)$, donde: $\mathrm{IS}=$ índice de sitio en metros, $\mathrm{Hd}=$ altura dominante, $\mathrm{b}=$ coeficiente de la ecuación de altura dominante, $\mathrm{Eb}=\mathrm{edad}$ base, $\mathrm{E}=$ edad de la plantación y $\mathrm{k}=$ exponente de mejor ajuste.

Los coeficientes resultantes de la regresión se listan a continuación:

\begin{tabular}{|c|c|c|c|c|}
\hline $\boldsymbol{b}_{\boldsymbol{1}}$ & $\boldsymbol{b}_{2}$ & $\mathrm{k}$ & $\mathrm{R}^{2}$ & $\mathrm{CME}$ \\
\hline 4,943007 & $-4,142972$ & 0,32 & 0,943056 & 0,512614 \\
\hline
\end{tabular}

* Instituto de Investigaciones de la Amazonía Peruana, Programa de Ecosistemas Terrestres. Correo electrónico: eotarola@iiiap.org.pe 
La ecuación de índice de sitio permitió clasificar las plantaciones forestales en tres calidades o clases de sitio: alta (I), media (II) y baja (III), cuyo ámbito fue definido por la desviación estándar y el promedio de los valores de índice de sitio de todas las observaciones. Así, las parcelas cuyo índice de sitio es mayor a 28,04 m de altura dominante a una edad base de 15 años pertenecen a la calidad o clase de sitio alta; la clase media está comprendida entre los valores 22,32 y $28,04 \mathrm{~m}$ y en la clase de sitio baja, se encuentran las parcelas con una altura dominante menor a $22,32 \mathrm{~m}$.

Palabras clave: Cedrelinga catenaeformis, índice de sitio, productividad, crecimiento, potencial de sitio, parcelas permanentes.

\section{ABSTRACT}

In the area of influence of the Jenaro Herrera Research Center (CIJH) was estimated the quality of the site based on the site indexes for the forest species Cedrelinga catenaeformis Ducke «tornillo». 1349 tree species from 29 permanent sample plots were evaluated. These plots consist of 25 a 49 individual trees distributed in 15 silvicultural experimental-type forest plantations.

Site index trend estimation were built using the dominant height of plots at different ages from 0,3 to 27 years. The 15 -year age of plantation was chosen as the most representative in the data base. Site index trends were improved according to the methodology described by Clutter et al. (1983).

The dominant height performance related to age was estimated using the Schumacher model $\left.[\mathrm{Ln}(\mathrm{Hd})]=b_{1}+b_{2}(1 / \mathrm{E})^{\mathrm{k}}\right]$. Equation formula for site index based on age and dominant height was estimated using the Hugell guide curve method (1991), by simplifying the previous equation into $\operatorname{Ln}(\mathrm{IS})=\operatorname{Ln}(\mathrm{Hd})+\mathrm{b}\left(1 / \mathrm{Eb}^{\mathrm{k}}-1 / \mathrm{E}^{\mathrm{k}}\right)$, where IS = site index in meters, $\mathrm{Hd}=$ dominant height, $\mathrm{b}=$ coefficient for dominant height, $\mathrm{Eb}=$ basis age, $\mathrm{E}=$ plantation age, $\mathrm{k}=$ improved adjustment exponent.

Resulting regression coefficients are listed as follows:

\begin{tabular}{|c|c|c|c|c|}
\hline $\boldsymbol{b}_{\boldsymbol{1}}$ & $\boldsymbol{b}_{\mathbf{2}}$ & $\mathrm{k}$ & $\mathrm{R}^{2}$ & $\mathrm{CME}$ \\
\hline 4,943007 & $-4,142972$ & 0,32 & 0,943056 & 0,512614 \\
\hline
\end{tabular}

The site index equation allowed to classify forest plantations into three site qualities: high (I), medium (II) and low (III); where standard deviation and site index average records of all observations determined the area of influence. Thus, plots with site 
index higher than $28,04 \mathrm{~m}$ are classify as high quality, from 22,32 to 28,04 as medium quality and plots with site index lower than 22,32 are classify as low quality.

This work is part of a great approach research aimed at to establish a relationship among growth and productivity of plantation with site indexes and physical and chemical factor of each plot, leading to better use of potential sites in Jenaro Herrera.

Key words: Cedrelinga catenaeformis, site index, productivity, growth, site potential, permanent plots.

\section{INTRODUCCIÓN}

El aprovechamiento selectivo de los bosques viene ocasionando la reducción de las poblaciones naturales de las especies forestales más valiosas, así como también tanto desabastecimiento de materia prima a las industrias como encarecimiento de productos. Frente a esta situación, una solución técnica es el establecimiento de plantaciones, ya sea para el enriquecimiento de bosques aprovechados, ya sea para repoblar áreas sin bosques.

El Centro de Investigaciones Jenaro Herrera (CIJH) cuenta actualmente con plantaciones de 32 especies, algunas de ellas con 29 años de evaluación permanente. Cedrelinga catenaeformis es una de las especies más estudiadas y sobresalientes. A los 15 años presentó incrementos anuales superiores a $1,5 \mathrm{~cm}$ de diámetro y $1,5 \mathrm{~m}$ de altura (Claussi et al., 1992). Su desarrollo y las bondades comerciales de la madera, tanto en el mercado nacional como en el internacional, hacen necesario profundizar estudios complementarios sobre el comportamiento de la especie en plantaciones forestales. En este sentido, la estimación de la calidad de sitio se hace más importante cuando se trata del manejo forestal intensivo, donde su campo de aplicación se extiende sobre la valoración de las tierras con aptitud forestal, el análisis económico de las operaciones silviculturales, la estimación del crecimiento y del rendimiento, los estudios del mejoramiento de los árboles y los estudios del mejoramiento del sitio, entre otros.

\section{Área de estudio}

\section{Ubicación}

Las plantaciones forestales que son materia de este estudio se encuentran ubicadas en los terrenos pertenecientes al Centro de Investigaciones Jenaro Herrera (CIJH), 
estación experimental del Instituto de Investigaciones de la Amazonía Peruana (IIAP). El CIJH se encuentra a $2,8 \mathrm{~km}$ del pueblo de Jenaro Herrera (4⿳⺈冂大 $\left.55^{\prime} \mathrm{S}, 73^{\circ} 44^{\prime} \mathrm{E}\right)$, perteneciente al distrito de Jenaro Herrera, provincia de Requena, departamento de Loreto.

\section{Clima}

En el CIJH la precipitación promedio anual fue de $2730 \mathrm{~mm}$ durante un periodo de observación ocurrido entre 1972 y 1994. Abril es el mes de mayor precipitación (294,3 mm) y julio el de menor (155,2 mm). El periodo más lluvioso corresponde a los meses comprendidos entre octubre y mayo (más de $200 \mathrm{~mm}$ mensuales). El lapso entre enero y abril es el más intenso dentro de este periodo. La época menos lluviosa está comprendida entre junio y setiembre.

La temperatura media anual durante el periodo $1972-1994$ fue de $26,9^{\circ} \mathrm{C}$ con una variación de $2^{\circ} \mathrm{C}$. Los promedios de las temperaturas mínima y máxima son de $21,1^{\circ} \mathrm{C}$ y $32,6^{\circ} \mathrm{C}$, respectivamente. La mayor temperatura se registra durante los meses de setiembre y octubre $\left(33,3^{\circ} \mathrm{C}\right.$ en ambos casos) y las menores en julio y agosto $\left(19,1^{\circ} \mathrm{C}\right.$ y $20,1^{\circ} \mathrm{C}$, respectivamente).

\section{Fisiografía}

La zona de Jenaro Herrera (López Parodi y Freitas, 1990) presenta dos paisajes bien diferenciados: la planicie aluvial fluviátil y la zona de altura (tierra firme). En la zona de altura o tierra firme, se identifican tres grandes unidades fisiográficas: la terraza baja, la terraza alta y la colina baja. Todas las plantaciones utilizadas para este estudio se localizan en terraza alta, la que se muestra como una extensa llanura, cruzada por valles de 6 a $12 \mathrm{~m}$ de profundidad y de 10 a $300 \mathrm{~m}$ de ancho. El fondo de los valles es plano. Estos se denominan localmente bajiales y son recorridos por quebradas que forman meandros y laderas con pendientes que superan el $10 \%$.

\section{Suelos}

Las plantaciones han sido instaladas sobre suelos amarillo-anaranjados de las llanuras y laderas de terraza alta. Estos se caracterizan por ser fuertemente ácidos (de 3,9 a 4,6 de $\mathrm{pH}$ ) y ostentar una textura que va de franco-areno-arcilloso a arcillo-arenoso sin fragmentos gruesos. Asimismo, estos suelos presentan una estructura granular con intensa presencia de raíces que penetran hasta una profundidad que oscila entre 60 y $70 \mathrm{~cm}$, por debajo de la cual la estructura se transforma en masiva. Algunos suelos presentan un horizonte superior grisáceo bien desarrollado (Claussi et al., 1992). 


\section{MATERIALES Y MÉTODOS}

En el área de influencia del Centro de Investigaciones Jenaro Herrera, se realizó la estimación de la calidad de sitio a partir de «índices de sitio» para la especie Cedrelinga catenaeformis Ducke (tornillo), debido a que esta define en gran parte la productividad de los terrenos forestales expresada en volumen comercial cosechado. En este sentido, Ferreira (1995) acota que no es posible tomar decisiones válidas de tipo silvicultural si no se hace referencia a la calidad del sitio. La calidad del sitio expresa el crecimiento o productividad promedio de una especie en función de su capacidad de adaptación y respuesta a determinadas condiciones de clima y suelo, lo que se traduce en la capacidad de la especie para generar volumen en cantidad y calidad en el menor tiempo posible (Spurr, 1982, citado por Solorio y Manzanilla, 1993).

Para hacer posible este estudio, se realizaron mediciones multitemporales de 29 parcelas permanentes de crecimiento (PPC) instaladas en 15 plantaciones del tipo experimental silvicultural (1 349 árboles), cuyas superficies son de 0,25-1 ha. Estas se conformaron con la finalidad de desarrollar técnicas silviculturales adecuadas para su manejo. Las plantaciones están distribuidas en tres campos experimentales denominados como sigue (ver croquis): Campo Abierto Laurent, que comprende las plantaciones 103 (año 1975), 106 (año 1972), 111 (año 1971) y 112 (año 1975); Campo Abierto El Piñal, que comprende las plantaciones 202 (año 1973), 204 (año 1973), 209 (año 1974), 210 (año 1975), 213 (año 1975), 216 (año 1974) y 218 (año 1985); Campo Abierto El Cañal, que comprende las plantaciones 403 (año 1973), las plantaciones agroforestales 406 (año 1986), 504 (año 1977) y 510 (año 1988).

Se aprovechó la información histórica de las mediciones dasométricas que se realizaron desde el año 1973 hasta la actualidad. En estas se midieron los parámetros de diámetro a la altura del pecho (dap), diámetro a la altura del suelo (base de la planta), altura total, altura al inicio de la copa, altura comercial, proyección de la copa, tendencia del árbol y estado fitosanitario. El número de mediciones por plantación fue variable y se reportan entre 6 y 9 mediciones realizadas en diferentes años.

Se construyeron curvas de índice de sitio utilizando la altura dominante de las parcelas a diferentes edades y el método conocido como de la «Curva Guía» (Alder, 1980), el cual es un procedimiento ideado para construir sistemas de curvas anamórficas de índices de sitio (Hugell, 1991). Se utilizó un amplio rango de edades que van desde 0,3 hasta 27 años. La edad base elegida fue 15 años por ser la más representativa en la base de datos. Se ajustaron las curvas de índices de sitio desarrollando la metodología descrita por Clutter et al. (1983). 
Los datos de campo fueron digitados en el programa Manejo de Información sobre Recursos Arbóreos, Componente de Silvicultura (MIRASILV) desarrollado por el CATIE. En este programa, se realizó el procesamiento preliminar de la información con la finalidad de obtener los valores promedio de las parcelas y seleccionar la información por procesar. Posteriormente, utilizando el módulo de estadística de Excel, se realizó el análisis estadístico de la información para las estimaciones y clasificación de los índices y calidad de sitio.

El índice de sitio se define como la altura dominante que pueden alcanzar los árboles de un rodal a una edad determinada, llamada edad base (Ugalde y Vásquez, 1995). A partir de esta edad, se estimó el comportamiento de la altura dominante de las parcelas a diferentes edades utilizando el modelo de Schumacher: $\left.[\mathrm{Ln}(\mathrm{Hd})]=b_{1}+b_{2}(1 / \mathrm{E})^{\mathrm{k}}\right]$; donde: $\mathrm{Ln}=$ Logaritmo natural, $b_{1}$ y $b_{2}=$ coeficientes y $\mathrm{k}=$ constante.

Los coeficientes resultantes de la regresión son los siguientes:

\begin{tabular}{|c|c|c|c|c|}
\hline $\boldsymbol{b}_{\boldsymbol{1}}$ & $\boldsymbol{b}_{2}$ & $\mathrm{k}$ & $\mathrm{R}^{2}$ & $\mathrm{CME}$ \\
\hline 4,943007 & $-4,142972$ & 0,32 & 0,943056 & 0,512614 \\
\hline
\end{tabular}

Para estimar el valor de k se probaron varios valores en las regresiones: desde 0,05 hasta 2. Se escogió el valor que arrojó el mayor coeficiente de determinación. El coeficiente de mejor ajuste (k) es igual a 0,32 para la especie Cedrelinga catenaeformis.

Para obtener la ecuación que estima el índice de sitio sobre la base de la edad y la altura dominante, se utilizó el método de la curva guía. Luego de obtener la ecuación de mejor ajuste a los datos disponibles, se construyeron las curvas de índice de sitio con el método de pendiente común o curvas anamórficas. La ecuación simplificada que resultó fue: $\operatorname{Ln}(\mathrm{IS})=\operatorname{Ln}(\mathrm{Hd})+b_{2}\left(1 / E^{k}-1 / E^{k}\right)$, donde: IS=índice de sitio en metros, $\mathrm{Hd}=$ altura dominante, $\mathrm{b}_{2}=$ coeficiente de la ecuación de altura dominante, $\mathrm{Eb}=\mathrm{edad}$ base, $\mathrm{E}=\mathrm{edad}$ de la plantación, $\mathrm{k}=$ exponente de mejor ajuste (Figura 1).

Despejando la altura dominante, se construyó la familia de curvas anamórficas (las que mejor ajustaron para la especie). Para ver su ajuste, las curvas límite o umbrales de clase, fueron ploteadas con los valores históricos de altura dominante de las parcelas con la finalidad de estratificarlas gráficamente por clase y observar su comportamiento en el tiempo. 


\section{RESULTADOS Y DISCUSIÓN}

Existe una notable correspondencia entre los incrementos medio anuales (IMA) y las clases de sitio definidas. Un bajo porcentaje de las parcelas observadas corresponde a una calidad o clase de sitio baja, lo que corrobora por qué la especie tornillo está considerada como una especie promisoria para el área de influencia del CIJH. Las clases de índice de sitio fueron definidas del siguiente modo: las parcelas cuyo ámbito de calidad de sitio (expresado en altura dominante) es mayor o igual que 28,04 m a la edad base de 15 años pertenecen a la clase de sitio alta (I); la clase media (II) comprende las parcelas cuya altura dominante a la edad base de 15 años se encuentra entre 22,33 y 28,03 m; y en la clase de sitio baja (III) se encuentran las parcelas cuya altura dominante a la edad base de 15 años está por debajo de los 22,32 $\mathrm{m}$ (Cuadro 1 y Figura 1).

Cuadro 1. Promedios de valores por clase de sitio para Cedrelinga catenaeformis en Jenaro Herrera, Loreto.

\begin{tabular}{|l|c|c|c|c|c|}
\hline Clase de sitio & $\begin{array}{c}\text { Ámbito } \\
\text { IS* }\end{array}$ & $\begin{array}{c}\text { IMA Prom hdom } \\
\text { (m/año) }\end{array}$ & $\begin{array}{c}\text { Desviación } \\
\text { estándar } \\
(\mathrm{m})\end{array}$ & $\begin{array}{c}\mathrm{N}^{\circ} \text { de } \\
\text { observ. }\end{array}$ & $(\%)$ \\
\hline Alta (I) & $>=28,04$ & 2,10 & 0,43 & 74 & 41,6 \\
Media (II) & $22,33-28,03$ & 1,63 & 0,09 & 81 & 45,5 \\
Baja (III) & $<=22,32$ & 1,43 & 0,16 & 23 & 12,9 \\
\hline
\end{tabular}

*IS = altura dominante en $\mathrm{m}$ a los 15 años de edad base.

Las parcelas permanentes instaladas en la plantación 218 no presentan, en promedio, un comportamiento histórico homogéneo respecto de la calidad de sitio expresada en altura dominante. Así, se clasifican en la calidad de sitio alta hasta los 5 años, edad probable en que la competencia comenzó a afectar el crecimiento de las mismas. La temprana edad en la que se manifestó la alta competencia se explica por un distanciamiento inicial de solo $2 \times 2$. A partir de ese momento, se evidenció una tendencia de incrementos negativa que llevó a la plantación a la clase III, aproximadamente a los nueve años. Este comportamiento es atribuible a un manejo silvicultural inadecuado para las condiciones y nivel de competencia, y no al sitio per se, que originalmente pertenecía a una clase alta (I). En la plantación se observó un marcado efecto de micrositio. De esta manera, se alcanzó una desviación estándar de $0,38 \mathrm{~m}$ entre los promedios de las parcelas permanentes (Foto 1 y Figura 1). 
Las parcelas correspondientes a la plantación 209 se encuentran, predominantemente, en una clase de sitio alta (I). Cabe resaltar aquí los valores extremos en la PPC 07, que presentó $35 \mathrm{~m}$ de altura dominante a los 14,6 años. El promedio de IMA de altura dominante de todas las parcelas de esta plantación es de 1,88 m/año. En ella, existe poco efecto de micrositio, pues entre las PPC hay una pequeña desviación estándar $(0,046 \mathrm{~m})$.

La plantación 210, que forma parte de la plantación de El Piñal, también pertenece a la clase de sitio alta (I), a pesar de que se encontraba, en el momento de la evaluación de campo, en estrés por alta competencia (de las curvas puede deducirse que a partir del año 15). El promedio de IMA de altura dominante es de 1,79 m/año. Se encontró una desviación estándar mayor a la anterior $(0,087 \mathrm{~m})$, sin embargo, puede decirse que el sitio, desde el punto de vista de calidad, es relativamente homogéneo.

Desde el punto de vista de crecimiento, la plantación 204 se encuentra en el umbral entre las clases alta y media, por ello presenta el promedio de IMA de altura dominante más bajo de la clase alta (I): 1,70 m/año. Ello se puede explicar por el estado sanitario deficiente (calificado en la observación de campo de regular a malo) de algunos individuos del rodal.

La única plantación de clase de sitio alta (I) que no pertenece al sector de El Piñal es la 406 (sector El Cañal, costado norte del campamento principal del CIJH). Esta plantación, inicialmente agroforestal, presenta muy buen promedio de IMA de altura dominante $(2,50 \mathrm{~m} / \mathrm{año})$ y un comportamiento de índice de sitio bastante consistente en el tiempo. Probablemente, existió una relación sinérgica con los cultivos agrícolas que la acompañaron inicialmente, lo que favoreció el desarrollo juvenil del tornillo. Este comportamiento positivo se relaciona directamente con el uso anterior del suelo (bosque primario) y con el poco tiempo transcurrido entre la corta y el establecimiento de la plantación. Además, se aprecia un adecuado manejo silvicultural en el campo, que permite obtener individuos con buena calidad de fuste.

La plantación en fajas, 504 del sector El Cañal, pertenece a la clase de sitio media (II) y presenta un promedio de IMA de altura dominante de $1,68 \mathrm{~m} /$ año con muy poca variabilidad (la desviación estándar es de $0,011 \mathrm{~m}$ ). La plantación fue históricamente representativa de esta clase de sitio, salvo en la última medición (año 21.7) en la que, probablemente, por falta de una adecuada apertura del dosel superior, bajó a la clase III.

Las plantaciones 103, 106, y 111 presentan los promedios de IMA de altura dominante más bajos de la clase de sitio II o media: 1,60 m/año para las dos primeras, y 
1,46 m/año para la última (el extremo inferior de esta clase). Estas pertenecen al sector Juan Laurent y manifiestan un comportamiento de índice de sitio bastante consistente en el tiempo. Sin embargo, presentan en común un deficiente manejo: sin raleo ni podas, en el primer caso, y restringido a raleos sanitarios en los dos últimos.

Las plantaciones 202, 213 y 216, pertenecientes al sector El Piñal, por el contrario, presentan los promedios de IMA en altura dominante más altos de esta clase de sitio (II) con valores 1,$75 ; 1,65$ y $1,69 \mathrm{~m} /$ año, respectivamente.

Las plantaciones 510, 403 y 112, pertenecientes a la clase o calidad de sitio III o baja, presentan los menores promedios de crecimiento de todo el ámbito del estudio. El valor extremo inferior, $1,27 \mathrm{~m} / \mathrm{año}$, se presentó en la plantación 403 , perteneciente al sector El Cañal. El bajo crecimiento del tornillo en esta plantación se explica, en gran medida, por el uso anterior del suelo que, en este caso, fue de intenso pastoreo, lo que ocasionó que se compactara, degradara y perdiera la capa orgánica (Claussi et al.,1992). La plantación 510, también de El Cañal, y la 112 del sector Juan Laurent presentan valores de IMA de 1,58 y 1,45 m/año, respectivamente.

Sin embargo, consideramos importante mencionar que existen otros factores que han influido en el desarrollo de las plantaciones. Tal es el caso, por ejemplo, de la cantidad insuficiente de luz y del aprovechamiento selectivo de alto impacto en el rodal original en la plantación 510, establecida en fajas de enriquecimiento (Foto 2).

En resumen, se observa que las plantaciones del sector El Piñal presentan, en promedio, mejores crecimientos, y varias de sus plantaciones se encuentran en la clase de sitio alta (I) o en el estrato superior de la clase media. La mayoría de plantaciones del sector Juan Laurent está representada en el estrato inferior de la clase media (II) y en la clase baja (III), lo que es un indicador de la no muy buena calidad de sitio de este sector. El sector El Cañal presenta una mayor variabilidad, que se encuentra representada en todas las clases de sitio, aunque presenta el valor extremo inferior de crecimiento en la plantación 403 (1,27 m/año).

La Figura 1 resume gráficamente el comportamiento histórico, respecto de calidad de sitio, de los promedios de altura dominante de las parcelas permanentes de crecimiento y de las plantaciones forestales que representan. En la misma gráfica, se encuentran ploteadas las curvas de índice de sitio, que representan los umbrales de clases de sitio para las plantaciones de Cedrelinga catenaeformis en el área de influencia del CIJH. 


\section{CONCLUSIONES}

Existe una fuerte correspondencia entre los datos de crecimiento y la clasificación por índice de sitio. Este hecho indica que este tipo de estratificación es aplicable y tiene uso práctico para la especie.

Del total de parcelas evaluadas en este estudio, sólo un 12,9\% pertenece a clase de índice de sitio baja. La mayor parte de las mismas se encuentra situada en las clases de sitio media y alta, lo que confirma a Cedrelinga catenaeformis como una especie promisoria para el área de influencia del CIJH. Sin embargo, esto se verificará con mayor precisión mediante el análisis de los factores edáficos y los parámetros de productividad que ya se vienen investigando.

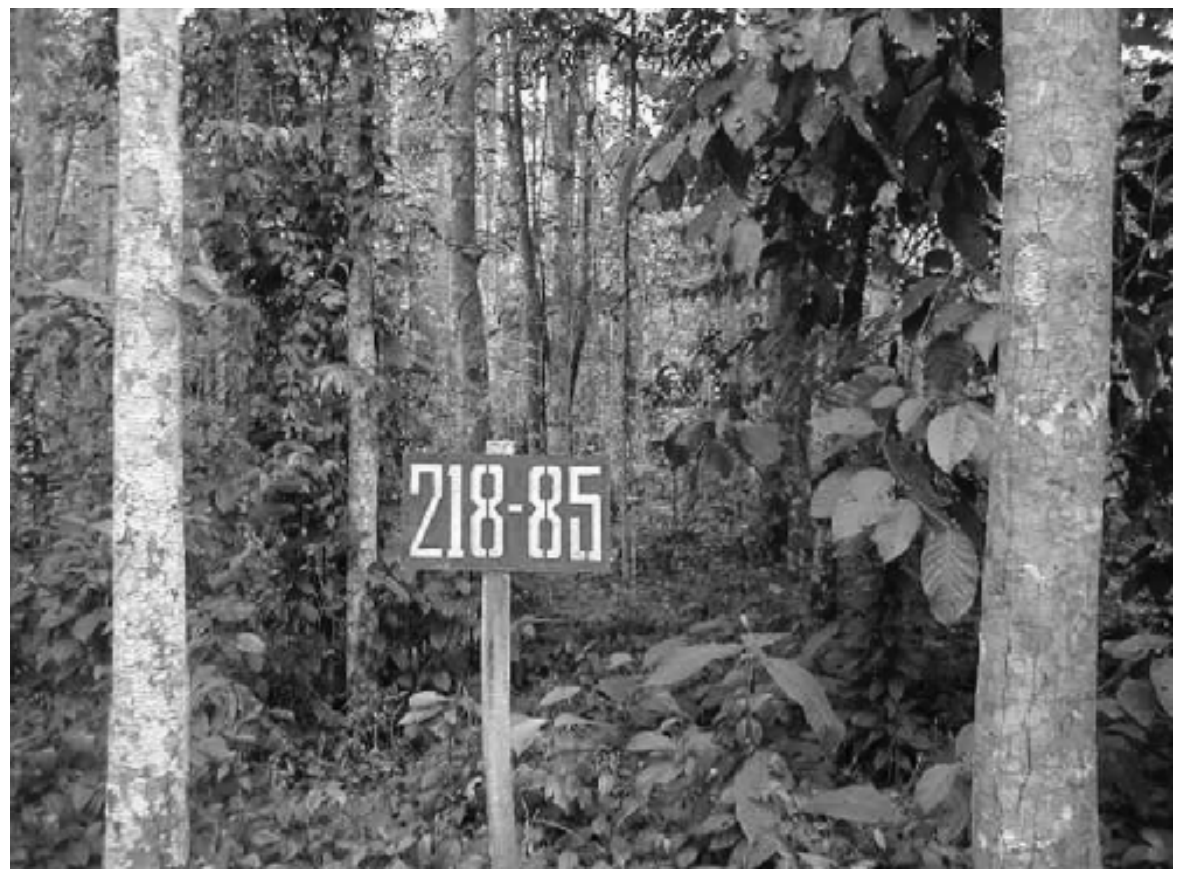

Foto 1. Plantación de Cedrelinga catenaeformis en una clase de sitio alta (I) a los 15 años de edad en el sector El Piñal, Centro de Investigaciones Jenaro Herrera, Loreto. 
Se identificaron como factores de alta sensibilidad en un modelo de crecimiento de Cedrelinga catenaeformis al uso anterior del suelo (la especie evidenció ser muy sensible a los suelos compactados) y al factor luz, especialmente en plantaciones en fajas de enriquecimiento.

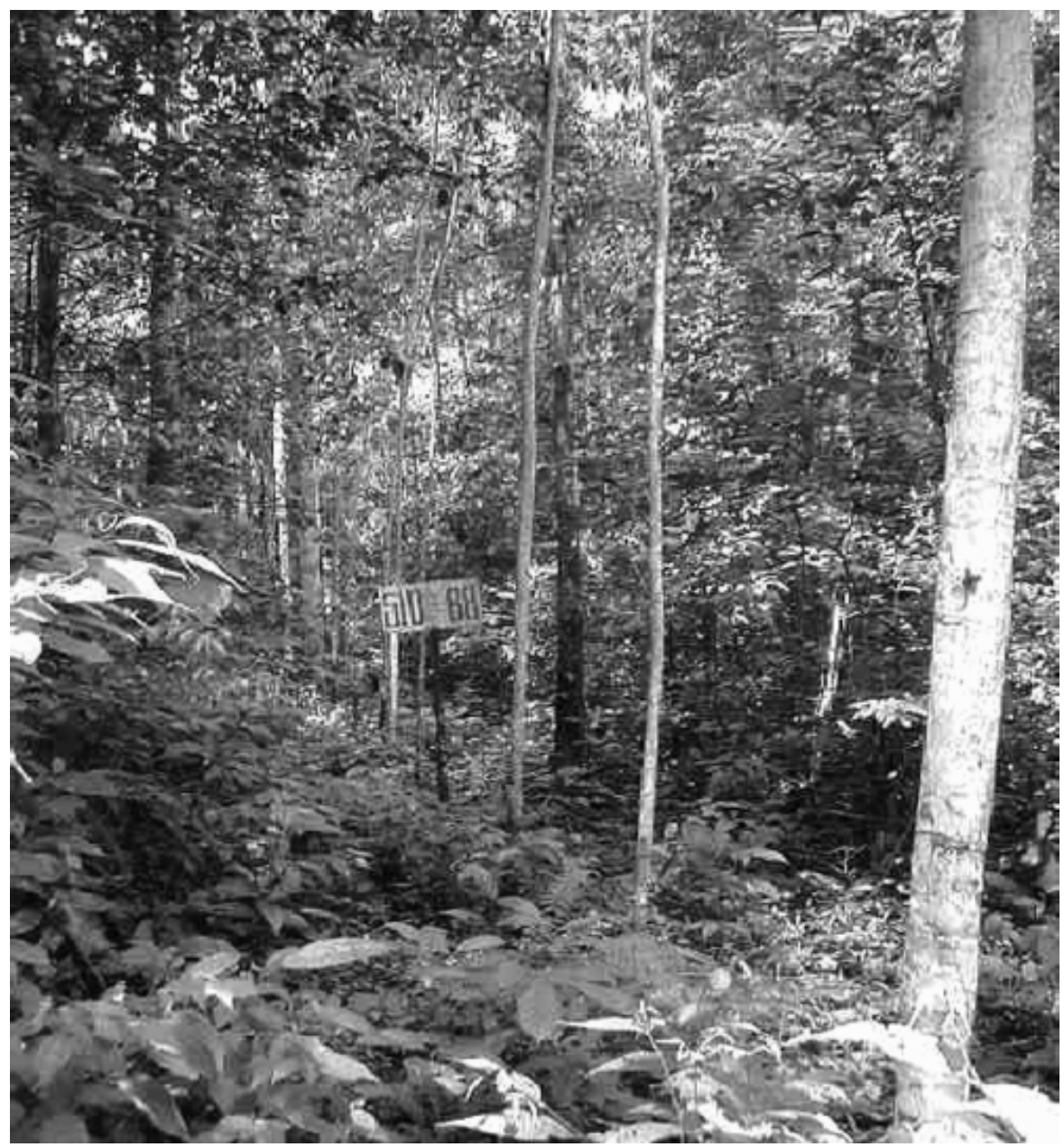

Foto 2. Plantación en fajas de Cedrelinga catenaeformis en una clase de sitio baja a los 12 años de edad en el sector El Cañal, Centro de Investigaciones Jenaro Herrera, Loreto. 


\section{BIBLIOGRAFÍA}

ALDER, D. 1980. Estimación del volumen forestal y predicción del rendimiento, con referencia especial a los trópicos. Roma (Italia): Estudios FAO. Vol. № 2. $197 \mathrm{pp}$.

CLAUSSI, A.; MARMILLOD, D.; BLASER, J. 1992. Descripción silvicultural de las plantaciones forestales de Jenaro Herrera. Instituto de Investigaciones de la Amazonía Peruana. Iquitos (Perú): Centro de Investigaciones Jenaro Herrera. 334 pp.

CLUTTER, R. L.; FORTOW, J. C.; PIENNAR, L. V.; BRISTER, G. H.; BAILEY, R. L. 1983. Timber Management: A Quantitative Approach. N. Y. (USA): J. Wiley. $31 \mathrm{pp}$.

FERREIRA, O. 1995. Manual de ordenación de Bosques. Siguatepeque (Honduras): CENIFA. $123 \mathrm{pp}$.

HUGELL, D. 1991. Modelos de predicción del crecimiento y rendimiento de: Gliricidia sepium, Guazuma ulmifolia y Leucaena leucocephala, en América Central. En: Serie Técnica No 22. Turrialba (Costa Rica): CATIE. $57 \mathrm{pp}$.

LÓPEZ-PARODI, J.; FREITAS, D. 1990. Geographical aspects of forested wetlands in the lower Ucayali, Peruvian Amazon. En: Forest Ecology and Management 33/34(1-4): 157-168.

SOLORIO, J.; MANZANILLA, H. 1993. Estimación de la calidad de sitio mediante índices de sitio de Pinus michoacana cornuta Martinez y Pinus Oocarpa Schiede para el A D F Tapalpa, Estado de Jalisco. En: Ciencia Forestal, Vol. 18(74).

UGALDE, L.; VÁSQUEZ, W. 1995. Rendimiento y calidad de sitio para Gmelina arborea, Tectona grandis, Bombacopsis quinatum y Pinus caribaea en Guanacaste. Informe Técnico. En: Serie Técnica $\mathrm{N}^{\circ}$ 256. Turrialba (Costa Rica): CATIE. 40 pp. 


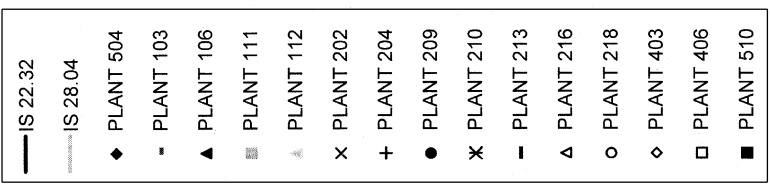

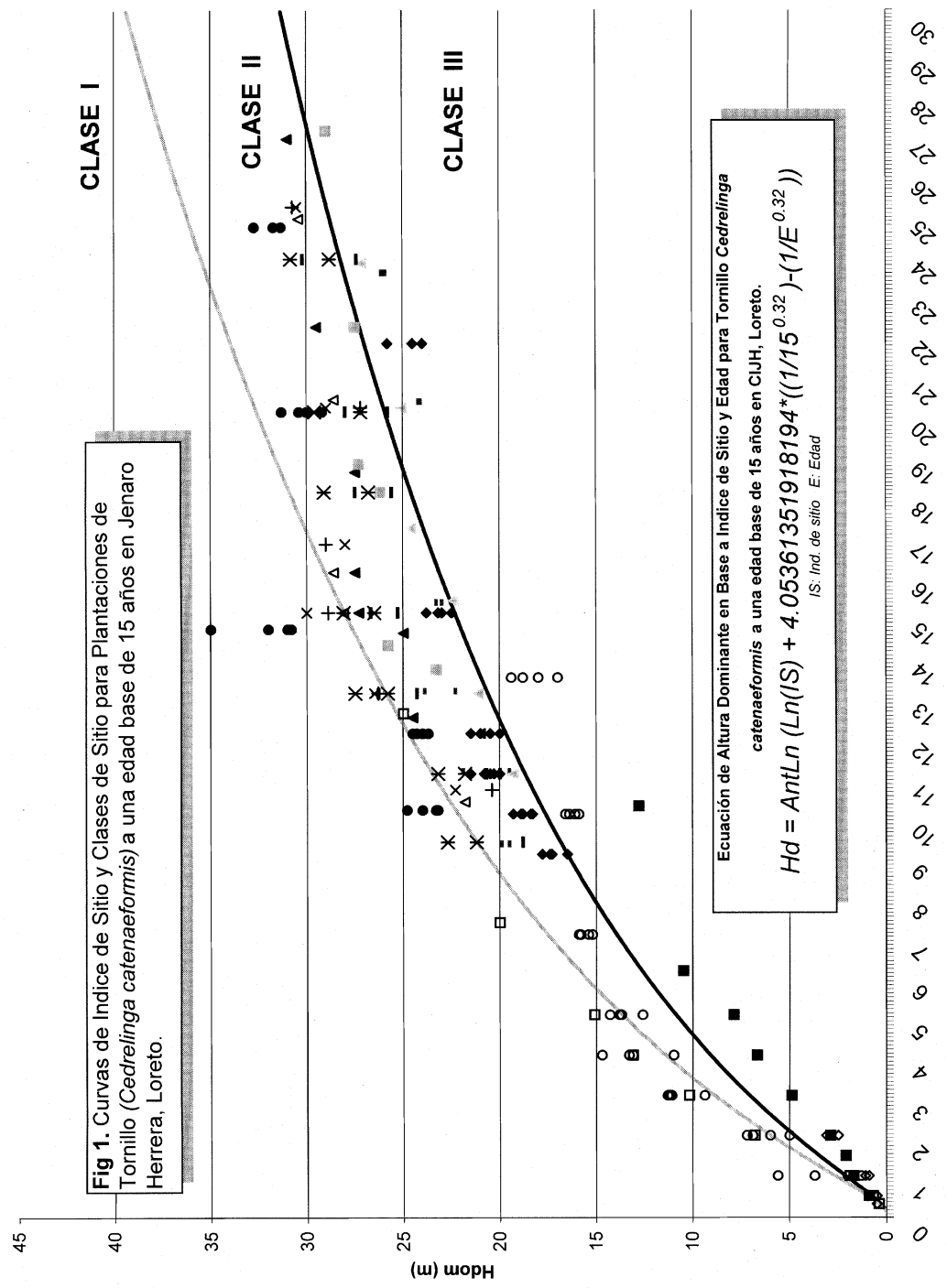

